\title{
Prestimulus and poststimulus cuing of recall order in the memory span
}

\section{JAMES V. HINRICHS, University of lowa, Iowa City, Iowa 52240}

Permutations of nine-digit sequences were recalled in either forward $(F)$ or backward $(B)$ order with order of recall cued either before the sequences (Prestimulus Cuing) or after the sequences (Poststimulus Cuing). Prestimulus Cuing produced significantly better recall than Poststimulus Cuing and $F$ recall was superior to $B$ recall, with a significant interaction between the two main effects. The shapes of the serial position curves for $F$ and $B$ recall were relatively unchanged by the position of cuing recall order, although the level of performance was lower in the Prestimulus Cuing condition.

In memory span recall, the $S$ typically is required to recall presented items in a designated order, usually the same order as presented. The required order is known to the $S$ before presentation and his attempted reproduction of that order results in the well-known bowed serial position curve. At least with forward (same order) recall, the observed serial position curve is relatively stable (Murdock, 1968). Backward (reverse order) recall also exhibits a typical bowed serial position curve. In forward recall, the primary effect predominates and in backward recall, the recency effect is greater. To what extent are these serial position effects dependent upon the S's prior knowledge of the required recall order? If knowledge of order of recall were not presented until after the to-be-remembered items were presented, would different serial position curves occur, possibly as a result of different behavioral strategies?

Poststimulus cuing has usually been used in memory studies to designate partial report of a larger set of presented items (Anderson, 1960; Sperling, 1960). The method has also been used to signal order of recall of sublists within a larger list (Kay \& Poulton, 1951) and can be adapted to indicate order of recall in testing immediate memory span. The present study compares forward and backward recall of digit sequences with either prestimulus or poststimulus cuing of the required recall order. Two questions are of particular interest. First, to what extent is recall performance dependent upon knowledge of the required recall order? Second, how are the serial position curves affected by poststimulus cuing of recall order?

\section{METHOD}

The Ss were 28 undergraduates fulfilling a course requirement. Four Ss failed to follow instructions and their data were not scored.

The Ss listened to two lists of 50 sequences. Each sequence consisted of a different permutation of the digits 1 through 9. The digits in each sequence were presented by a tape recorder at the rate of $1 / \mathrm{sec}$. The Ss recalled the sequences by writing the digits just presented on an answer sleet, placing each digit in a blank corresponding to its correct position. Twenty sec were allowed for recall.

For one list (Prestimulus Cuing), Ss were cued before presentation of the sequence whether recall was to be in the forward (F) or backward (B) order. The word "forward" or "backward" was spoken just before each sequence was presented. Forward recall required each digit to be recalled in the same order as it was presented: the first digit placed in the first answer blank. the second in the second blank, etc. Backward recall required each digit to be recalled in the reverse order of presentation; the last digit placed in the first blank, the next-to-last in the second blank. etc. Immediately after presentation of the last digit in the sequence, a brief tone signaled that recall was to begin. In each list, 25 sequences were to be recalled in the Forder and 25 in the $B$ order, with $F$ and $B$ orders randomly presented. In the Poststimulus Cuing condition, a second list of 50 sequences was presented, $25 \mathrm{~F}$ and $25 \mathrm{~B}$. but in this condition the tone signaled the start of presentation and the appropriate recall order. F or B. was presented immediately after the last digit was spoken and also served as the cue to start recall. The presentation of Prestimulus Cuing and Poststimulus Cuing conditions was counterbalanced across Ss as was the assignment of lists to each condition and the assignment of sequences to $F$ and $B$ recall orders. Thus, each sequence occurred in each of the four conditions an equal number of times.

In order to maintain the recall order manipulation, the Ss were required to fill in each answer blank in order. They were allowed to guess or to repeat responses, but each blank had to be completed before proceeding to the next. The Ss were not allowed to fill in blanks out of order, or to go back and change an answer after completing later items. A response was scored as correct only if the digits were in the correct serial position. RESULTS

The mean number of digits recalled in each condition was: with Prestimulus Cuing, $F=6.12, B=4.65$; with Poststimulus Cuing,
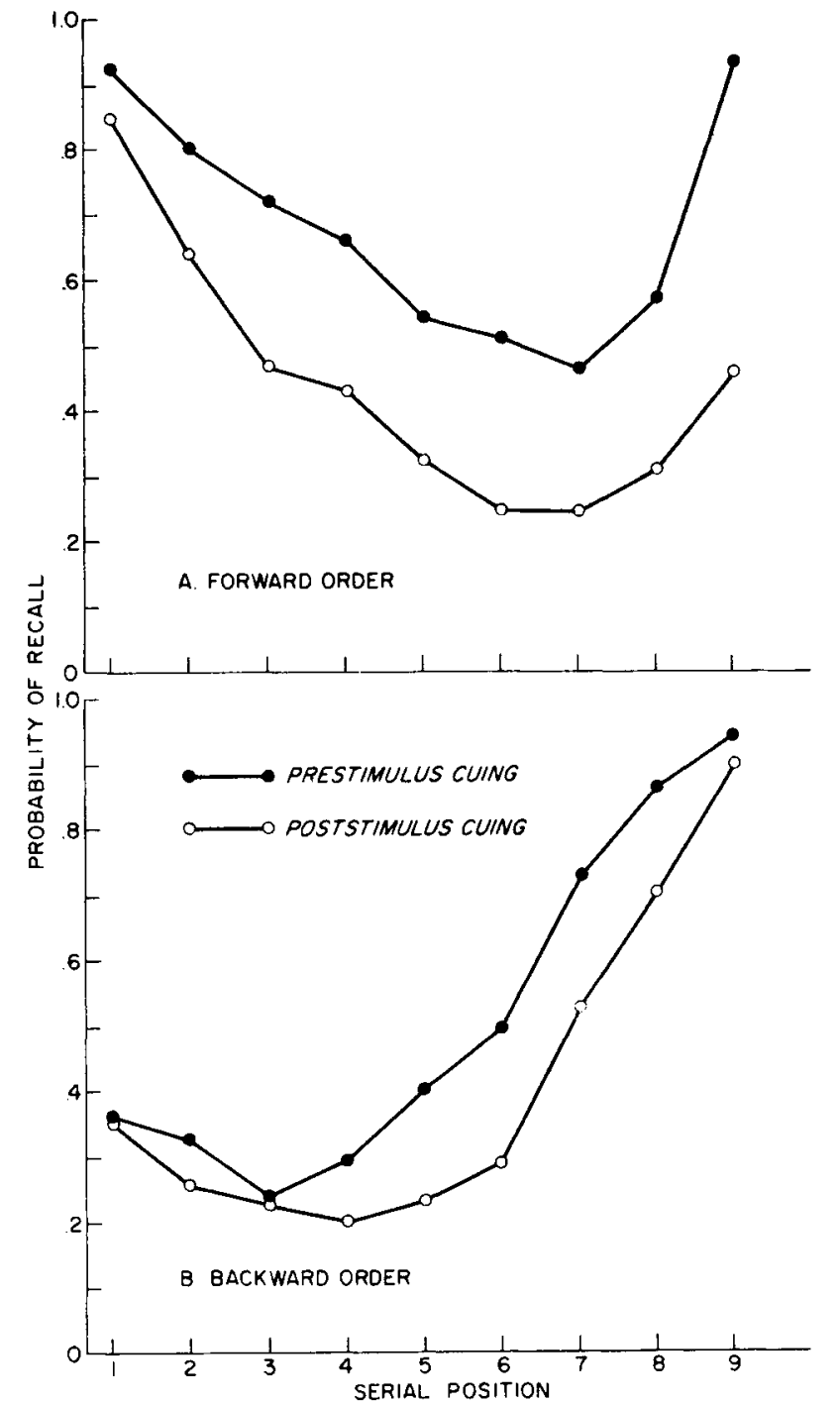

Fig. 1. Probability of recall in correct position as a function of presentation order with order of recall cued either before presentation (Prestimulus Cuing) or after presentation (Poststimulus Cuing): A. forward order recall; B. backward order recall. 
$\mathrm{F}=3.96, \mathrm{~B}=3.68$. Analysis of variance showed that both main effects, order of recall and position of cuing, and their interaction produced significant effects. Recall in the $F$ order was superior to the $B$ order $(F=46.23, \mathrm{df}=1 / 23, \mathrm{p}<.001)$. Prestimulus Cuing produced better performance than Poststimulus Cuing $(\mathrm{F}=154.91, \mathrm{df}=1 / 23, \mathrm{p}<.001)$. The Cuing by Recall Order interaction was significant $(F=33.39$, $\mathrm{df}=1 / 23, \mathrm{p}<.001)$, with the difference between $F$ and $B$ recall significant in the Prestimulus Cuing condition $(\mathrm{t}=8.08, \mathrm{df}=23, \mathrm{p}<.001)$ but not significant in the Poststimulus Cuing condition $(t=1.53, \mathrm{df}=23)$.

Prestimulus Cuing and Poststimulus Cuing serial position curves for the two recall orders are shown in Fig. 1. Within each recall order the serial position curves are approximately the same shape. The Poststimulus Cuing curves are below the Prestimulus Cuing curves but the functions are almost parallel. The $F$ curves (Panel A) show the usual bowed serial position with a moderately sloped primacy function and a short but steep recency curve. The only prominent difference between the Prestimulus Cuing and the Poststimulus Cuing curves other than the level of performance is the reduced recency effect in the Poststimulus Cuing curve. The B curves (Panel B) show a marked recency effect and very little primacy effect, but again no obvious differences in the shapes of the two curves are apparent.

\section{DISCUSSION}

When Ss know the required recall order before presentation they are able to recall in the conventional $F$ order far better than in the $\mathrm{B}$ order as measured by the mean number of digits recalled in the correct position. When the recall order is not known until after stimulus presentation, however, $F$ recall is not significantly superior to $B$ recall. Perhaps the superiority of $F$ recall is not surprising on the basis of prior immediate memory experience such as recalling telephone numbers, responding to conversation, and so forth, but then the failure of this experience to transfer to the Poststimulus Cuing condition is puzzling. If the superior performance in $\mathrm{F}$ order recall in the Prestimulus Cuing condition is to be attributed to transfer of recall habits and strategies, then it would be expected that these same habits and strategies would transfer to the Poststimulus Cuing situation and produce the same $F$ recall superiority.

Perhaps, as is often suggested, Ss approach different recall tasks with different rehearsal strategies. The different rehearsal strategies might be expected then to produce changes in the observed serial position curves. Certainly this interpretation could be used to account for the observed large differences in the serial position curves in $F$ and $B$ recall. If this were the case, then the Poststimulus Cuing condition would pose a great problem to the $S$. Either a new strategy would have to be invoked to meet the unknown recall order or else a recall strategy suitable for either $F$ or B recall would be chosen with the expectation that it would be inappropriate $50 \%$ of the time. In either case, if rehearsal strategies do affect the shape of the serial position curve a change in the curve would be expected. But this was not the case: the shape of the recall curves seemed to be relatively unaffected by the position of recall order cuing. Either serial position curves do not reflect Ss rehearsal strategies or else the rehearsal strategies did not differ with $F$ or B recall orders. In either case, it seems clear that the shape of the serial position curve is influenced more by the order in which items are recalled than by the knowledge of the required recall order. The absolute level of the serial position curve, and hence the overall mean recall performance, however, is raised by prior knowledge of the required recall order.

$$
\text { REFERENCES }
$$

ANDERSON, N. S. Poststimulus cuing in immediate memory. Journal of Experimenal Psychology, 1960, 60, 216-221.

KAY, H., \& POULTON, E. C. Anticipation in memorizing. British Journal of Psychology, 1951, 42, 34-41.

MURDOCK, B. B., Jr., Serial order effects in short-term memory. Journal of Experimental Psychology, 1968, 76 (Monograph Supplement).

SPERLING, G. The information available in brief visual presentations. Psychological Monographs, 1960, 74 (Whole No. 498). 